\title{
A Central Limit Theorem for the Volumes of High Excursions of Stationary Associated Random Fields
}

\author{
Vadim Demichev ${ }^{1,2}$, Judith Sarah Olszewski ${ }^{3, *}$ \\ ${ }^{1}$ Lomonosov Moscow State University, 119991 Moscow, Russia. ${ }^{2}$ The University of Sheffield, S10 2TN Sheffield, UK. \\ ${ }^{3}$ Ulm University, Institute of Stochastics, 89069 Ulm, Germany.
}

Received: 13 March 2015; Accepted: 27 March 2015

Editor: David G. Yu

\begin{abstract}
We prove that under certain conditions the excursion sets volumes of stationary positively associated random fields converge after rescaling to the normal distribution as the excursion level and the size of the observation window grow. In addition, we provide a number of examples.
\end{abstract}

Keywords Association, Excursion Set, Central Limit Theorem, Gaussian Random Field, Shot-Noise

DOI: $10.19139 /$ soic.v3i2.137

\section{Introduction}

The study of excursion set properties plays an increasingly important role within modern theory of random fields. Such objects arise in connection to a wide range of stochastic models (see, e.g., [1, 2]). Recently a number of works appeared $([3,4,5])$ focused on asymptotic properties of the excursion sets volumes corresponding to fixed excursion levels and sequences of growing (in a certain sense) observation windows. Spodarev gives in [6] an overview of the recent asymptotic results concerning the geometry of excursion sets of stationary associated random fields.

In this paper, we show that under certain conditions the excursion sets volumes of stationary positively associated random fields converge after rescaling to the normal distribution as both the excursion level and the size of the observation window grow. Note that a similar model was studied in the monograph of Ivanov and Leonenko [7] for the case of a Gaussian random field. For associated random fields on lattices the asymptotic behaviour of excursion sets cardinalities corresponding to a growing excursion level was examined in [8].

The present paper is organized as follows. In section 2 we provide the necessary definitions. Section 3 contains the main theorem. In section 4 we show how its conditions can be verified for Gaussian random fields, fields with regularly varying tails and shot-noise random fields.

\section{Preliminaries}

We assume that all random objects are defined on a complete probability space $(\Omega, \mathcal{F}, \mathbb{P})$.

*Correspondence to: Judith Sarah Olszewski (Email: judith.olszewski@uni-ulm.de). Ulm University, Institute of Stochastics, Helmholtzstraße 18, 89069 Ulm, Germany.

ISSN 2310-5070 (online) ISSN 2311-004X (print)

Copyright (C) 2015 International Academic Press 
Consider a family of random variables $\mathrm{X}=\left\{X_{t}, t \in T\right\}$. For $I \subset T$ set $X_{I}=\left\{X_{t}, t \in I\right\}$. Let $M(d)$ denote the class of real-valued bounded coordinate-wise nondecreasing Borel functions on $\mathbb{R}^{d}, d \in \mathbb{N}$. According to [9], $\mathrm{X}$ is called positively associated ( $\mathrm{X} \in \mathrm{PA}$ ) or weakly associated, if for arbitrary disjoint finite sets $I, J \subset T$ and any functions $f \in \mathcal{M}(\operatorname{card}(I)), g \in \mathcal{M}(\operatorname{card}(J))$ one has

$$
\operatorname{Cov}\left(f\left(X_{I}\right), g\left(X_{J}\right)\right) \geqslant 0 .
$$

Here $\operatorname{card}(I)$ denotes the cardinality of $I$. It is known that quite a wide range of random fields possesses the property of positive association (see [10]). For example, any Gaussian random field with nonnegative covariance function is positively associated.

Let $A_{u}(\mathrm{X}, T)=\left\{t \in T: X_{t} \geqslant u\right\}$ be the excursion set of the random field $\mathrm{X}=\left\{X_{t}, t \in \mathbb{R}^{d}\right\}$ at the level $u \in \mathbb{R}$ corresponding to the observation window $T \subset \mathbb{R}^{d}$ and let $v_{d}(B)$ be the volume of measurable $B \subset \mathbb{R}^{d}$. By $\mathbb{I}(C)$ we denote the indicator function of the set $C$. Obviously,

$$
V_{u}(\mathrm{X}, T)=v_{d}\left(A_{u}(\mathrm{X}, T)\right)=\int_{T} \mathbb{I}\left(X_{t} \geqslant u\right) d t .
$$

Let $\|\cdot\|$ and $\|\cdot\|_{\infty}$ be, respectively, the Euclidean norm and the maximum norm in $\mathbb{R}^{d}$ and $\mathcal{O}(\cdot)$ - the Landau notation.

\section{Central Limit Theorem}

Let $\mathrm{X}$ be a measurable strictly stationary positively associated random field on $\mathbb{R}^{d}$. We also assume that $\mathrm{X}$ is square integrable and its covariance function $r(t)=\operatorname{Cov}\left(X_{0}, X_{t}\right), t \in \mathbb{R}^{d}$, is continuous. Note that due to the latter condition $X$ is also associated (see [10] for the definition of association and related dependence types).

For any $u \in \mathbb{R}$ and bounded measurable $B, C \subset \mathbb{R}^{d}$ Fubini's theorem implies

$$
\begin{gathered}
\mathbb{E} V_{u}(\mathrm{X}, B)=v_{d}(B) \mathbb{P}\left(X_{0} \geqslant u\right), \\
\left.\left.\operatorname{Cov}\left(V_{u}(\mathrm{X}, B)\right), V_{u}(\mathrm{X}, C)\right)\right)=\int_{B} \int_{C} \operatorname{Cov}\left(\mathbb{I}\left(X_{s} \geqslant u\right), \mathbb{I}\left(X_{t} \geqslant u\right)\right) d s d t \geqslant 0 .
\end{gathered}
$$

Consider an increasing sequence of excursion levels $\left\{u_{n}\right\}_{n \in \mathbb{N}}, 0<u_{n} \rightarrow \infty, n \rightarrow \infty$. Define $S_{n}=V_{u_{n}}\left(\mathrm{X},[0, n)^{d}\right)$, $n \in \mathbb{N}$, and let $\sigma_{n}^{2}=\operatorname{Var} S_{n}$.

Suppose that the random variable $X_{0}$ has a bounded density $f(\cdot)$ and set $\gamma(x)=\sup _{t \geqslant x} f(t), x \in \mathbb{R}$.

\section{Theorem 1}

Assume that the random field $\mathrm{X}$ satisfies the following conditions:

(A1) $r(t)=\mathcal{O}\left(\|t\|^{-\mu}\right)$ for some $\mu>3 d$;

(A2) $\sigma_{n} \rightarrow \infty, n \rightarrow \infty$;

(A3) $\delta_{n}=n^{d} \gamma^{2 / 3}\left(u_{n}\right) \sigma_{n}^{-(1+\mu / 3 d)} \rightarrow 0, n \rightarrow \infty$.

Then

$$
\frac{S_{n}-\mathbb{E} S_{n}}{\sqrt{\operatorname{Var} S_{n}}} \stackrel{d}{\longrightarrow} \mathcal{N}(0,1),
$$

where $\stackrel{d}{\longrightarrow}$ denotes convergence in distribution.

The following lemma can be proved in the same way as Lemma 7.3.4 from [10].

\section{Lemma 1}

Under the conditions of Theorem 1

$$
\operatorname{Cov}\left(\mathbb{I}\left(X_{s} \geqslant u\right), \mathbb{I}\left(X_{t} \geqslant u\right)\right) \leqslant 3 \cdot 2^{2 / 3} \gamma^{2 / 3}(u) r^{1 / 3}(s-t), \quad u \in \mathbb{R}, s, t \in \mathbb{R}^{d} .
$$


Proof of Theorem 1. One can find a sequence $m_{n} \rightarrow \infty, n \rightarrow \infty$, such that $m_{n}^{d} / \sigma_{n} \rightarrow 0$ and

$$
\frac{n^{d}}{\sigma_{n}^{2}} \gamma^{2 / 3}\left(u_{n}\right) m_{n}^{d-\mu / 3} \longrightarrow 0, \quad n \rightarrow \infty
$$

To see this, consider $m_{n}=\max \left\{\sigma_{n}^{1 /(2 d)}, \delta_{n}^{1 /(\mu-3 d)} \sigma_{n}^{1 / d}\right\}$.

Set $q_{n}=\sigma_{n}^{1 /(2 d)} m_{n}^{1 / 2}$ and $r_{n}=\left\lfloor\frac{n}{q_{n}}\right\rfloor$. Note that

$$
\frac{m_{n}}{q_{n}} \longrightarrow 0, \quad \frac{q_{n}^{d}}{\sigma_{n}} \longrightarrow 0, \quad n \rightarrow \infty .
$$

Using (1), (A1) and (2), we also get $\sigma_{n}^{2}=\mathcal{O}\left(n^{d}\right), n \rightarrow \infty$. Hence, $r_{n} \rightarrow \infty$ as $n \rightarrow \infty$. Clearly, there exists $N_{0} \in \mathbb{N}$ such that $0<m_{n}^{d} \leqslant q_{n}^{d} \leqslant \sigma_{n}$ for all $n>N_{0}$.

Define

$$
\widetilde{S}_{n}=V_{u_{n}}\left(\mathrm{X},\left[0, r_{n} q_{n}\right)^{d}\right)=\sum_{k \in \mathbb{Z}^{d} \cap\left[0, r_{n}\right)^{d}} \zeta_{n, k}, \quad Z_{n}=\sum_{k \in \mathbb{Z}^{d} \cap\left[0, r_{n}\right)^{d}} \xi_{n, k},
$$

where $\zeta_{n, k}=V_{u_{n}}\left(\mathrm{X},\left[0, q_{n}\right)^{d} \oplus k q_{n}\right), k \in \mathbb{Z}^{d} \cap\left[0, r_{n}\right)^{d}$, and $\left\{\xi_{n, k}, k \in \mathbb{Z}^{d} \cap\left[0, r_{n}\right)^{d}\right\}$ are independent random variables distributed in the same way as $V_{u_{n}}\left(\mathrm{X},\left[0, q_{n}\right)^{d}\right), n>N_{0}$. Approximating integrals with finite sums in $\mathrm{L}_{1}$ (see [4]) it is not difficult to show that, since the random field $\mathrm{X}$ is positively associated and its covariance function is continuous, $\left\{\zeta_{n, k}, k \in \mathbb{Z}^{d} \cap\left[0, r_{n}\right)^{d}\right\} \in \mathrm{PA}$.

Before we continue with the proof of the theorem, we establish the following lemma.

\section{Lemma 2}

Under the conditions of Theorem 1 it holds that

$$
\frac{\operatorname{Var} Z_{n}}{\operatorname{Var} S_{n}} \longrightarrow 1, \quad n \rightarrow \infty
$$

Proof. Due to (1), the variance of $Z_{n}$ is not greater than the variance of $S_{n}$. Thus, it suffices to prove that

$$
\limsup _{n \rightarrow \infty} \frac{\operatorname{Var} S_{n}}{\operatorname{Var} Z_{n}} \leqslant 1
$$

Observe that for $n>N_{0}$

$$
\begin{aligned}
\operatorname{Var} S_{n} & =\operatorname{Var} Z_{n}+\sum_{k \in \mathbb{Z}^{d} \cap\left[0, r_{n}\right)^{d}} \operatorname{Cov}\left(V_{u_{n}}\left(\mathrm{X},\left[0, q_{n}\right)^{d} \oplus k q_{n}\right), V_{u_{n}}\left(\mathrm{X},[0, n)^{d} \backslash\left(\left[0, q_{n}\right)^{d} \oplus k q_{n}\right)\right)\right) \\
& +\operatorname{Cov}\left(V_{u_{n}}\left(\mathrm{X},[0, n)^{d} \backslash\left[0, r_{n} q_{n}\right)^{d}\right), V_{u_{n}}\left(\mathrm{X},[0, n)^{d}\right)\right)=\operatorname{Var} Z_{n}+\Sigma_{1}+\Sigma_{2} .
\end{aligned}
$$

Below we show that $\Sigma_{1}$ and $\Sigma_{2}$ admit the following estimates

$$
\begin{gathered}
\Sigma_{1} \leqslant C_{1} n^{d} \gamma^{2 / 3}\left(u_{n}\right) m_{n}^{d-\mu / 3}+C_{2} \frac{m_{n}}{q_{n}} \operatorname{Var} Z_{n}, \\
\Sigma_{2} \leqslant C_{3} r_{n}^{-1} \operatorname{Var} S_{n}, \quad n>N_{0} .
\end{gathered}
$$

Here, and in the following, $C_{1}, C_{2}, C_{3}, \ldots$ are some positive real numbers which may only depend on $d, r$ and $\mu$. Applying (6) and (7), we have

$$
\frac{\operatorname{Var} S_{n}}{\operatorname{Var} Z_{n}}\left(1-C_{3} r_{n}^{-1}-C_{1} \frac{n^{d}}{\sigma_{n}^{2}} \gamma^{2 / 3}\left(u_{n}\right) m_{n}^{d-\mu / 3}\right) \leqslant 1+C_{2} \frac{m_{n}}{q_{n}}, \quad n>N_{0} .
$$

Due to (3) and (4), the latter inequality implies (5). 
Now let us obtain (6). It can be easily seen that (1) yields

$$
\begin{aligned}
& \operatorname{Cov}\left(V_{u_{n}}\left(\mathrm{X},\left[0, q_{n}\right)^{d} \oplus k q_{n}\right), V_{u_{n}}\left(\mathrm{X},[0, n)^{d} \backslash\left(\left[0, q_{n}\right)^{d} \oplus k q_{n}\right)\right)\right) \\
& \leqslant \operatorname{Cov}\left(V_{u_{n}}\left(\mathrm{X},\left[0, q_{n}\right)^{d}\right), V_{u_{n}}\left(\mathrm{X},[-n, 2 n)^{d} \backslash\left[0, q_{n}\right)^{d}\right)\right), k \in \mathbb{Z}^{d} \cap\left[0, r_{n}\right)^{d} .
\end{aligned}
$$

Thus, $\Sigma_{1} \leqslant R_{1}+R_{2}$, where

$$
\begin{aligned}
& R_{1}=r_{n}^{d} \operatorname{Cov}\left(V_{u_{n}}\left(\mathrm{X},\left[0, q_{n}\right)^{d}\right), V_{u_{n}}\left(\mathrm{X},[-n, 2 n)^{d} \backslash\left[-m_{n}, q_{n}+m_{n}\right)^{d}\right)\right), \\
& R_{2}=r_{n}^{d} \operatorname{Cov}\left(V_{u_{n}}\left(\mathrm{X},\left[0, q_{n}\right)^{d}\right), V_{u_{n}}\left(\mathrm{X},\left[-m_{n}, q_{n}+m_{n}\right)^{d} \backslash\left[0, q_{n}\right)^{d}\right)\right) .
\end{aligned}
$$

Using (2) and (A1), we get

$$
\begin{aligned}
R_{1} & \leqslant C_{4} \gamma^{2 / 3}\left(u_{n}\right) r_{n}^{d} q_{n}^{d} \int_{\|x\|_{\infty}>m_{n}} r^{1 / 3}(x) d x \\
& \leqslant C_{5} \gamma^{2 / 3}\left(u_{n}\right) n^{d} \int_{\|x\|_{\infty}>m_{n}}\|x\|^{-\mu / 3} d x \leqslant C_{1} n^{d} \gamma^{2 / 3}\left(u_{n}\right) m_{n}^{d-\mu / 3} .
\end{aligned}
$$

Now we show that $R_{2} \leqslant C_{2}\left(m_{n} / q_{n}\right) \operatorname{Var} Z_{n}$. Set $\tau=\left\lfloor\frac{2 q_{n}}{m_{n}}\right\rfloor$. Clearly,

$$
\begin{aligned}
& \frac{m_{n}}{q_{n}} \operatorname{Var} Z_{n}=\frac{m_{n}}{q_{n}} r_{n}^{d} \operatorname{Var} V_{u_{n}}\left(\mathrm{X},\left[0, q_{n}\right)^{d}\right) \geqslant C_{6} \frac{m_{n}}{q_{n}} r_{n}^{d} \operatorname{Var} V_{u_{n}}\left(\mathrm{X},\left[-2 q_{n}, 3 q_{n}\right)^{d}\right) \\
\geqslant & C_{6} \frac{m_{n}}{q_{n}} r_{n}^{d} \operatorname{Cov}\left(V_{u_{n}}\left(\mathrm{X},\left[-2 q_{n}, 3 q_{n}\right)^{d}\right), V_{u_{n}}\left(\mathrm{X},\left[-2 q_{n}, 3 q_{n}\right)^{d} \backslash\left[0, q_{n}\right)^{d}\right)\right) \\
\geqslant & C_{6} \frac{m_{n}}{q_{n}} r_{n}^{d} \operatorname{Cov}\left(V_{u_{n}}\left(\mathrm{X},\left[-2 q_{n}, 3 q_{n}\right)^{d}\right),\right. \\
& \left.\sum_{i=1}^{\tau} V_{u_{n}}\left(\mathrm{X},\left[-i m_{n}, q_{n}+i m_{n}\right)^{d} \backslash\left[-(i-1) m_{n}, q_{n}+(i-1) m_{n}\right)^{d}\right)\right) \\
\geqslant & C_{6} \frac{m_{n}}{q_{n}} r_{n}^{d} \sum_{i=1}^{\tau} \operatorname{Cov}\left(V_{u_{n}}\left(\mathrm{X},\left[-(i-1) m_{n}, q_{n}+(i-1) m_{n}\right)^{d}\right),\right. \\
& \left.V_{u_{n}}\left(\mathbf{X},\left[-i m_{n}, q_{n}+i m_{n}\right)^{d} \backslash\left[-(i-1) m_{n}, q_{n}+(i-1) m_{n}\right)^{d}\right)\right) \\
\geqslant & C_{6} r_{n}^{d} \operatorname{Cov}\left(V_{u_{n}}\left(\mathbf{X},\left[0, q_{n}\right)^{d}\right), V_{u_{n}}\left(\mathbf{X},\left[-m_{n}, q_{n}+m_{n}\right)^{d} \backslash\left[0, q_{n}\right)^{d}\right)\right) .
\end{aligned}
$$

Hence, the desired inequality holds for $C_{2}=1 / C_{6}$. To conclude the proof of the lemma, it remains to note that the method we used to estimate $R_{2}$ also yields (7). Lemma 2 is proved.

Due to (7) and Lemma 2 the terms $\frac{S_{n}-\mathbb{E} S_{n}}{\sqrt{\operatorname{Var} S_{n}}}$ and $\frac{\widetilde{S}_{n}-\mathbb{E} \widetilde{S}_{n}}{\sqrt{\operatorname{Var} Z_{n}}}$ have the same limiting distribution as $n \rightarrow \infty$. Thus, it suffices to show that $\frac{\widetilde{S}_{n}-\mathbb{E} \widetilde{S}_{n}}{\sqrt{\operatorname{Var} Z_{n}}}$ has the same limiting distribution as $\frac{Z_{n}-\mathbb{E} Z_{n}}{\sqrt{\operatorname{Var} Z_{n}}}$ and that $\frac{Z_{n}-\mathbb{E} Z_{n}}{\sqrt{\operatorname{Var} Z_{n}}}$ converges in distribution to the standard Gaussian law as $n \rightarrow \infty$.

Applying Newman's inequality (cf. [10, Corollary 1.5.5]), we get

$$
\begin{aligned}
& \left|\mathbb{E} \exp \left(i t \frac{\widetilde{S}_{n}-\mathbb{E} \widetilde{S}_{n}}{\sqrt{\operatorname{Var} Z_{n}}}\right)-\mathbb{E} \exp \left(i t \frac{Z_{n}-\mathbb{E} Z_{n}}{\sqrt{\operatorname{Var} Z_{n}}}\right)\right| \\
& \leqslant \sum_{\substack{k, l \in \mathbb{Z}^{d} \cap\left[0, r_{n}\right)^{d} \\
k \neq l}} \frac{2 t^{2}}{\operatorname{Var} Z_{n}} \operatorname{Cov}\left(V_{u_{n}}\left(\mathrm{X},\left[0, q_{n}\right)^{d} \oplus k q_{n}\right), V_{u_{n}}\left(\mathrm{X},\left[0, q_{n}\right)^{d} \oplus l q_{n}\right)\right) \\
& \leqslant \frac{2 t^{2}}{\operatorname{Var} Z_{n}} \Sigma_{1},
\end{aligned}
$$


where $\Sigma_{1}$ appears in the proof of Lemma 2. Now it is implied by (A2), (6), (3), Lemma 2 and (4) that the right-hand side of (8) tends to zero as $n \rightarrow \infty$.

As for the convergence of $\frac{Z_{n}-\mathbb{E} Z_{n}}{\sqrt{\operatorname{Var} Z_{n}}}$ to $\mathcal{N}(0,1)$, it follows from the central limit theorem in the form of Lindeberg. Indeed, the Lindeberg function

$$
L_{n}(\varepsilon)=\frac{1}{\operatorname{Var} Z_{n}} \sum_{k \in \mathbb{Z}^{d} \cap\left[0, r_{n}\right)^{d}} \mathbb{E}\left(\xi_{n, k}-\mathbb{E} \xi_{n, k}\right)^{2} \mathbb{I}\left(\left|\xi_{n, k}-\mathbb{E} \xi_{n, k}\right|>\varepsilon \sqrt{\operatorname{Var} Z_{n}}\right)
$$

is equal to zero for any $\varepsilon>0$ and $n>n_{0}(\varepsilon)$, since for all $k$

$$
\frac{\left|\xi_{n, k}-\mathbb{E} \xi_{n, k}\right|}{\sqrt{\operatorname{Var} Z_{n}}} \leqslant \frac{q_{n}^{d}}{\sqrt{\operatorname{Var} Z_{n}}} \longrightarrow 0, \quad n \rightarrow \infty .
$$

In order to obtain (9), we employed (4) and Lemma 2. The other conditions of the Lindeberg theorem are also easily checked. Theorem 1 is proved.

\subsection{Gaussian Random Fields}

Let $\mathrm{X}=\left\{X_{t}, t \in \mathbb{R}^{d}\right\}$ be a measurable stationary positively associated Gaussian random field with $\mathbb{E} X_{0}=0$ and $\operatorname{Var} X_{0}=1$. Suppose that the covariance function $r(\cdot)$ is continuous and fulfills (A1). Consider

$$
u_{n} \leqslant \sqrt{c \log n}, \quad c=\frac{d(\mu-3 d)}{(1+\nu)(\mu+d)}, \quad n \in \mathbb{N},
$$

where $\nu$ is some positive number. For any $s, t \in \mathbb{R}^{d}$ it holds (see [4]) that

$$
\operatorname{Cov}\left(\mathbb{I}\left(X_{s} \geqslant u_{n}\right), \mathbb{I}\left(X_{t} \geqslant u_{n}\right)\right)=\frac{1}{2 \pi} \int_{0}^{r(t-s)} \frac{1}{\sqrt{1-x^{2}}} \exp \left(-\frac{u_{n}^{2}}{1+x}\right) d x, n \in \mathbb{N} .
$$

Consequently,

$$
\operatorname{Var} S_{n} \geqslant \frac{1}{2 \pi} \exp \left(-u_{n}^{2}\right) \int_{[0, n)^{d}} \int_{[0, n)^{d}} r(t-s) d t d s .
$$

Since $r(\cdot)$ is continuous and $r(0)=1$, we have

$$
\liminf _{n \rightarrow \infty} \frac{\operatorname{Var} S_{n}}{n^{d} \exp \left(-u_{n}^{2}\right)}>0 .
$$

It is easy to show that (10) implies (A2). Applying (10) and the fact that

$$
\gamma\left(u_{n}\right)=\frac{1}{\sqrt{2 \pi}} \exp \left(-u_{n}^{2} / 2\right)
$$

we also obtain

$$
\delta_{n}=\mathcal{O}\left(n^{-(\mu-3 d) / 6} \exp \left(\frac{1}{6}(1+\mu / d) u_{n}^{2}\right)\right), n \rightarrow \infty .
$$

It is easily seen that for our choice of $c$

$$
\frac{n^{c(1+\mu / d)}}{n^{\mu-3 d}} \longrightarrow 0 \text { as } n \rightarrow \infty
$$

Therefore, $\delta_{n} \rightarrow 0$ as $n \rightarrow \infty$.

The case of a Gaussian random field with non-integrable covariance function was considered by Ivanov and Leonenko in [7]. It is interesting to note that the central limit theorem they obtained features the sequence $\left\{u_{n}, n \in \mathbb{N}\right\}$ growing rapidly enough for Var $S_{n}$ to converge to 0 as $n \rightarrow \infty$. 


\subsection{Random Fields with Regularly Varying Tails}

Let $\mathrm{X}=\left\{X_{t}, t \in \mathbb{R}^{d}\right\}$ be a measurable stationary square integrable positively associated random field. Assume that its covariance function $r(\cdot)$ belongs to the $C^{2}\left(\mathbb{R}^{d}\right)$ class and fulfills (A1). We also assume that $X_{0}$ has a bounded density. Let $F$ be the distribution function of $X_{0}$. Suppose that it admits, for some $\alpha>0$, the following representation

$$
1-F(x)=x^{-\alpha} L(x), x>0,
$$

where $L(\cdot)$ is a slowly varying function on $(0, \infty)$, i.e. $1-F(\cdot)$ is regularly varying in the sense of Karamata (see [11]). We will assume $\alpha>2$. Note that for every $\varepsilon>0$

$$
x^{\varepsilon} L(x) \longrightarrow \infty, \quad \frac{L(x)}{x^{\varepsilon}} \longrightarrow 0, \quad x \rightarrow \infty .
$$

Since $r(\cdot)$ attains its maximum at zero, $\nabla r(0)=0$. Therefore, using Taylor's expansion, one can find $b>0$ and $\delta \in(0,1 / 2)$ such that

$$
r(0)-r(t) \leqslant b\|t\|^{2} \text { for all } t \in[0, \delta)^{d} .
$$

We show that under certain restrictions on the growth rate of $u_{n}, n \rightarrow \infty, \mathrm{X}$ satisfies the conditions of Theorem 1 . We need to estimate the following covariance from below

$$
\operatorname{Cov}\left(\mathbb{I}\left(X_{0} \geqslant u_{n}\right), \mathbb{I}\left(X_{t} \geqslant u_{n}\right)\right), \quad t \in[0, \delta)^{d}, \quad n \in \mathbb{N} .
$$

Clearly,

$$
\begin{aligned}
& \operatorname{Cov}\left(\mathbb{I}\left(X_{0} \geqslant u_{n}\right), \mathbb{I}\left(X_{t} \geqslant u_{n}\right)\right)=\mathbb{P}\left(X_{0} \geqslant u_{n}, X_{t} \geqslant u_{n}\right)-\mathbb{P}^{2}\left(X_{0} \geqslant u_{n}\right) \\
& \geqslant \mathbb{P}\left(X_{0} \geqslant 2 u_{n}, X_{0}-X_{t} \leqslant u_{n}\right)-\mathbb{P}^{2}\left(X_{0} \geqslant u_{n}\right) \\
& \geqslant \mathbb{P}\left(X_{0} \geqslant 2 u_{n}\right)-\mathbb{P}\left(X_{0}-X_{t} \geqslant u_{n}\right)-\mathbb{P}^{2}\left(X_{0} \geqslant u_{n}\right), \quad n \in \mathbb{N} .
\end{aligned}
$$

Using Markov's inequality, (12) and the fact that $2(r(0)-r(t))=\mathbb{E}\left(X_{0}-X_{t}\right)^{2}$, we obtain

$$
\begin{aligned}
& \mathbb{P}\left(X_{0} \geqslant 2 u_{n}\right)-\mathbb{P}\left(X_{0}-X_{t} \geqslant u_{n}\right)-\mathbb{P}^{2}\left(X_{0} \geqslant u_{n}\right) \\
& \geqslant 2^{-\alpha} u_{n}^{-\alpha} L\left(2 u_{n}\right)-2 b\|t\|^{2} u_{n}^{-2}-u_{n}^{-2 \alpha} L^{2}\left(u_{n}\right) \\
& =\Delta_{1}(n)-\Delta_{2}(n)-\Delta_{3}(n), \quad t \in[0, \delta)^{d}, \quad n \in \mathbb{N} .
\end{aligned}
$$

Applying (11), it is easy to show that for arbitrary $\varepsilon>0$

$$
\frac{2 b u_{n}^{-\alpha-2 \varepsilon}+\Delta_{3}(n)}{\Delta_{1}(n)} \longrightarrow 0, \quad n \rightarrow \infty .
$$

Hence, for sufficiently large $n$ and $\|t\| \leqslant u_{n}^{1-\alpha / 2-\varepsilon}$ we have

$$
\Delta_{1}(n)-\Delta_{2}(n)-\Delta_{3}(n) \geqslant \frac{1}{2} \Delta_{1}(n) \geqslant A u_{n}^{-\alpha-\nu}
$$

for any $\nu>0$ and some $A=A(L, \alpha, \nu)>0$.

Therefore, for any $\varepsilon, \nu>0$ and large enough $n$, it holds that

$\int_{[0, \delta)^{d}} \operatorname{Cov}\left(\mathbb{I}\left(X_{0} \geqslant u_{n}\right), \mathbb{I}\left(X_{t} \geqslant u_{n}\right)\right) d t \geqslant \int_{\left[0, u_{n}^{1-\alpha / 2-\varepsilon}\right)^{d}} \operatorname{Cov}\left(\mathbb{I}\left(X_{0} \geqslant u_{n}\right), \mathbb{I}\left(X_{t} \geqslant u_{n}\right)\right) d t \geqslant A u_{n}^{d-(d+2) \alpha / 2-\varepsilon d-\nu}$.

Consequently,

$$
\sigma_{n}^{2} \geqslant(n / 2)^{d} \int_{[0, \delta)^{d}} \operatorname{Cov}\left(\mathbb{I}\left(X_{0} \geqslant u_{n}\right), \mathbb{I}\left(X_{t} \geqslant u_{n}\right)\right) d t \geqslant A 2^{-d} n^{d} u_{n}^{d-(d+2) \alpha / 2-\varepsilon d-\nu} .
$$


Thus, $\sigma_{n} \rightarrow \infty$ if $u_{n}=\mathcal{O}\left(n^{a}\right), n \rightarrow \infty$, where $a<d /((d+2) \alpha / 2-d)$.

Since the density of $X_{0}$ is bounded, we can always find $c \geqslant 0$ such that

$$
\gamma(x)=\mathcal{O}\left(x^{-c}\right), x \rightarrow \infty .
$$

We have

$$
\begin{aligned}
\delta_{n} & =n^{d} \gamma^{2 / 3}\left(u_{n}\right) \sigma_{n}^{-(1+\mu /(3 d))} \\
& \leqslant A_{2} u_{n}^{-2 c / 3} n^{d / 2-\mu / 6} u_{n}^{(1+\mu /(3 d))((d+2) \alpha / 2-d+\varepsilon d+\nu) / 2} \\
& \leqslant A_{2} n^{-1 / 6(\mu-3 d)} n^{\max \{0,-2 a c / 3+a(\mu+3 d)((d+2) \alpha / 2-d+\varepsilon d+\nu) /(6 d)\}},
\end{aligned}
$$

where $A_{2}>0$ does not depend on $n$. It is not difficult to show that $\delta_{n} \rightarrow 0, n \rightarrow \infty$, if

$$
a<\frac{2 d(\mu-3 d)}{\max \{0,(\mu+3 d)((d+2) \alpha-2 d)-8 c d\}},
$$

where $s / 0=\infty, s \in \mathbb{R}$. Note that if

$$
f(x)=x^{-\alpha-1} L_{1}(x), x>0,
$$

where $f$ is the density of $X_{0}$ and $L_{1}$ is a slowly varying function on $(0, \infty)$, then $c$ can be taken to be any nonnegative number less than $\alpha+1$. Consequently, $\delta_{n} \rightarrow 0, n \rightarrow \infty$, provided

$$
a<\frac{2 d(\mu-3 d)}{\max \{0,(\mu+3 d)((d+2) \alpha-2 d)-8 d(\alpha+1)\}} .
$$

\subsection{Shot-Noise Random Fields}

In this section, the requirements of Theorem 1 are checked for certain shot-noise random fields and for the excursion level $u_{n}=\mathcal{O}\left((\log n)^{1-\beta}\right), n \rightarrow \infty$, where $\beta \in(0,1)$. The following definition is taken from [12].

Let $\phi$ be a stationary Poisson counting measure with intensity $\lambda>0$. Also, let $g: \mathbb{R} \rightarrow \mathbb{R}$ be a deterministic function with $\int_{\mathbb{R}} g(x) d x<\infty$ and $\int_{\mathbb{R}} g^{2}(x) d x<\infty$. The random field $\mathrm{X}=\left\{X_{t}, t \in \mathbb{R}\right\}$ defined by

$$
X_{t}=\int_{\mathbb{R}} g(t-x) \phi(d x), \quad t \in \mathbb{R},
$$

is called a shot-noise random field. The function $g$ is called a response function.

Here we consider shot-noise random fields with the intensity $\lambda>1 / 2$ and the response function

$$
g(t)=\exp (-|t|), t \in \mathbb{R} .
$$

In this case, $\mathrm{X}$ is square integrable and for any $\mu>3$ (A1) is fulfilled.

The characteristic function $\varphi_{X_{0}}(\cdot)$ of $X_{0}$ is (see [10, Lemma 1.3.7]) given by

$$
\varphi_{X_{0}}(s)=\exp \left(\lambda \int_{\mathbb{R}}\left(e^{i s e^{-|t|}}-1\right) d t\right), \quad s \in \mathbb{R} .
$$

It is not difficult to show that for $\lambda>1 / 2 \varphi_{X_{0}}(\cdot)$ is integrable and, consequently, $X_{0}$ has a bounded density.

In addition, one can show that the distribution of $X_{0}$ is infinitely divisible (see, e.g., [13]) with Lévy measure

$$
\psi(d x)=2 \lambda / x \mathbb{I}(x \in(0,1)) d x .
$$

In order to find an approximation for the distribution of $X_{0}$, we consider upper records. Let $\left\{Z_{n}: n \in \mathbb{N}\right\}$ be a sequence of independent and identically distributed observations. The observation $Z_{j}, j \in \mathbb{N}$, is called an upper record, if its value exceeds all previous observations, that is $Z_{j}>\max \left\{Z_{1}, Z_{2}, \ldots, Z_{j-1}\right\}$. Since the distribution 
of $X_{0}$ is infinitely divisible and the Lévy measure $\psi$ has a density $l$ on $[0, \infty)$ with $\int_{0}^{\infty} l(y) d y=\infty$, it follows from [14, Theorem 2] that

$$
(n+1)-T_{n} \longrightarrow X_{0} \text { a.s., }
$$

where $T_{n}, n \in \mathbb{N}$, is the sum of the first $(n+1)$ upper records from a certain distribution $H$. Moreover, the density of $H$ is known and, in our case, is given by

$$
h(x)=2 \lambda(1-x)^{2 \lambda-1} \mathbb{I}(x \in(0,1)), \quad x \in \mathbb{R} .
$$

Thus, $H$ is the distribution function of a Beta-distributed random variable with parameters $2 \lambda$ and 1 . Such Beta records are considered in [15]. There it is shown that

$$
\lim _{n \rightarrow \infty}(n+1)-T_{n} \stackrel{d}{=} \sum_{i=0}^{\infty} \prod_{j=0}^{i} U_{j}^{\frac{1}{2 \lambda}}
$$

where $U_{j}, j \in \mathbb{Z}_{+}$, are independent random variables distributed uniformly on $(0,1)$.

Vervaat showed in [16, Theorem 4.7.7 and Lemma 4.7.9] that the density $f(x)$ of the random variable in the right-hand side of (14) (which is also the density of $X_{0}$ ) is nonincreasing, for sufficiently large $x$, and that

$$
f(x)=\exp (-(1+o(1)) x \log x), x \rightarrow \infty .
$$

To check the requirements of Theorem 1, the following result from [17] is used.

Let $\mathbf{N}=\mathbf{N}(\mathbb{R})$ be the space of integer-valued $\sigma$-finite measures $\kappa$ on $\mathbb{R}$ and let $\mathcal{N}$ be the smallest $\sigma$-algebra making the mappings $\kappa \rightarrow \kappa(B), \kappa \in \mathbf{N}$, measurable for all $B \in \mathcal{B}(\mathbb{R})$, where $\mathcal{B}\left(\mathbb{R}^{d}\right)$ is the Borel $\sigma$-algebra of $\mathbb{R}^{d}$, $d \in \mathbb{N}$. For a measurable $\psi: \mathbf{N} \rightarrow \mathbb{R}$ define

$$
D_{y} \psi(\kappa)=\psi\left(\kappa+\delta_{y}\right)-\psi(\kappa), \kappa \in \mathbf{N},
$$

where $\delta_{y}$ is the Dirac measure at $y \in \mathbb{R}$. For $k \in \mathbb{N}$ and $\left(y_{1}, \ldots, y_{k}\right) \in \mathbb{R}^{k}$ set

$$
D_{y_{1}, \ldots, y_{k}}^{k} \psi=D_{y_{1}}^{1}\left(D_{y_{2}, \ldots, y_{k}}^{k-1} \psi\right),
$$

where $D^{1}=D$ and $D^{0} \psi=\psi$. Define

$$
R_{k} \psi\left(y_{1}, \ldots, y_{k}\right)=\mathbb{E} D_{y_{1}, \ldots, y_{k}}^{k} \psi(\phi),
$$

if this expectation exists, where $\phi$ appears in (13). In [17, Theorem 1.1] it is shown that for any $\psi, \eta \in L_{2}\left(\mathbf{N}, \mathbb{P}_{\phi}\right)$, where $\mathbb{P}_{\phi}$ is the distribution of $\phi$ in $(\mathbf{N}, \mathcal{N})$, the following relation holds

$$
\mathbb{E} \psi(\phi) \eta(\phi)=\mathbb{E} \psi(\phi) \mathbb{E} \eta(\phi)+\sum_{k=1}^{\infty} \frac{1}{k !}\left\langle R_{k} \psi, R_{k} \eta\right\rangle_{L_{2}\left(\mathbb{R}^{k}, \lambda^{k} v_{k}\right)} .
$$

Here, $v_{k}$ is the Lebesgue measure on $\left(\mathbb{R}^{k}, \mathcal{B}\left(\mathbb{R}^{k}\right)\right), k \in \mathbb{N}$.

Define $\psi_{n}(\phi)=\int_{[0, n)} \mathbb{I}\left(X_{t} \geqslant u_{n}\right) d t$. In order to establish (A2), it is sufficient to show that

$$
\left\langle R_{1} \psi_{n}, R_{1} \psi_{n}\right\rangle_{L_{2}\left(\mathbb{R}, \lambda v_{1}\right)}=\lambda \int_{\mathbb{R}}\left(\int_{0}^{n} \mathbb{P}\left(u_{n}-e^{-|t-x|} \leqslant X_{t}<u_{n}\right) d t\right)^{2} d x \rightarrow \infty, \quad n \rightarrow \infty .
$$

Applying Vervaat's result, it holds that, for sufficiently large $n$,

$$
\int_{\mathbb{R}}\left(\int_{0}^{n} \mathbb{P}\left(u_{n}-e^{-|t-x|} \leqslant X_{t}<u_{n}\right) d t\right)^{2} d x \geqslant f^{2}\left(u_{n}\right) \int_{\mathbb{R}}\left(\int_{0}^{n} e^{-|t-x|} d t\right)^{2} d x .
$$


Therefore,

$$
\left\langle R_{1} \psi_{n}, R_{1} \psi_{n}\right\rangle_{L_{2}\left(\mathbb{R}, \lambda v_{1}\right)} \geqslant \lambda f^{2}\left(u_{n}\right)(n-2) .
$$

Since $u_{n}=\mathcal{O}\left((\log n)^{1-\beta}\right), n \rightarrow \infty, \beta \in(0,1),(15)$ and (17) imply (16).

It remains to show that $\delta_{n} \rightarrow 0, n \rightarrow \infty$. For $n$ large enough, the inequality (17) yields

$$
\delta_{n}=n \gamma^{\frac{2}{3}}\left(u_{n}\right) \sigma_{n}^{-(1+\mu / 3)} \leqslant A \frac{n\left(f\left(u_{n}\right)\right)^{\frac{2}{3}}}{\left(f^{2}\left(u_{n}\right) n\right)^{\frac{1}{6}(\mu+3)}}=A n^{-\frac{1}{6}(\mu-3)}\left(f\left(u_{n}\right)\right)^{-\frac{1}{3}(\mu+1)},
$$

where $A=A(\lambda)>0$. It is easy to see that the right-hand side of (18) converges to zero as $n \rightarrow \infty$.

\section{Acknowledgement}

The authors wish to thank Professor E. Spodarev for posing the problem and attention to this research. The authors are also grateful to Professor A. V. Bulinski for useful comments and encouragement. In addition, thanks are due to the Lomonosov Moscow State University and the University of Ulm for allowing this collaboration to happen.

\section{REFERENCES}

1. J. M. Azaïs and M. Wschebor, Level Sets and Extrema of Random Processes and Fields, Wiley, New York, 2009.

2. R. J. Adler and J. E. Taylor, Random Fields and Geometry, Springer Monographs in Mathematics, Springer, New York, 2007.

3. D. Meschenmoser and A. Shashkin, Functional central limit theorem for the volume of excursion sets generated by associated random fields, Statistics and Probability Letters, vol. 81, no. 6, pp. 642-646, 2011.

4. A. Bulinski, E. Spodarev and F. Timmermann, Central limit theorems for the excursion sets volumes of weakly dependent random fields, Bernoulli, vol. 18, no. 1, pp. 100-118, 2012.

5. V. Demichev, Functional central limit theorem for excursion set volumes of quasi-associated random fields, Journal of Mathematical Sciences, vol. 204, no. 1, pp. 69-77, 2015.

6. E. Spodarev, Limit theorems for excursion sets of stationary random fields, In Modern Stochastics and Applications, Springer Optimization and its Applications, vol. 90, pp. 221-244, 2014.

7. A. V. Ivanov and N. N. Leonenko, Statistical Analysis of Random Fields, Kluwer, Dordrecht, 1989.

8. Y. Bakhtin, Poisson limit for associated random fields, Theory of Probability and its Applications, vol. 54, no. 4, pp. 678-681, 2010.

9. C. M. Newman, Asymptotic independence and limit theorems for positively and negatively dependent random variables, in Inequalities in Statistics and Probability, Lecture Notes-Monograph Series, Institute of Mathematical Statistics, vol. 5, pp. 127-140, 1984.

10. A. Bulinski and A. Shashkin, Limit Theorems for Associated Random Fields and Related Systems, World Scientific, Singapore, 2007.

11. J. Karamata, Sur un mode de croissance régulière. Théorèmes fondamentaux, Bulletin de la Société Mathématique de France, vol. 61, pp. 55-62, 1933.

12. A. Bulinski and E. Spodarev, Introduction to random fields, in Stochastic Geometry, Spatial Statistics and Random Fields. Asymptotic Methods, Lecture Notes in Mathematics, Springer, Berlin, vol. 2068, pp. 277-335, 2013.

13. K. Sato, Lévy Processes and Infinitely Divisible Distributions, Cambridge Studies in Advanced Mathematics, Cambridge University Press, 2007.

14. A. Bose, S. Gangopadhyay, A. Sarkar and A. Sengupta, Asymptotic properties of sums of upper records, Extremes, vol. 6, pp. 147-164, 2003.

15. B. C. Arnold and J. A. Villaseñor, The asymptotic distributions of sums of records, Extremes, vol. 1, no. 3, pp. 351-363, 1999.

16. W. Vervaat, Success Epochs in Bernoulli Trials, with Applications in Number Theory, Mathematisch Centrum, Amsterdam, 1972.

17. G. Last and M. D. Penrose, Poisson process Fock space representation, chaos expansion and covariance inequalities, Probability Theory and Related Fields, Springer, Berlin, vol. 150, pp. 663-690, 2011. 